\title{
Electrically conductive pili from pilin genes of phylogenetically diverse microorganisms
}

\author{
David JF Walker ${ }^{1}$, Ramesh Y Adhikari ${ }^{2,4,5}$, Dawn E Holmes ${ }^{1,3}$, Joy E Ward ${ }^{1}$, \\ Trevor L Woodard ${ }^{1}$, Kelly P Nevin ${ }^{1}$ and Derek R Lovley ${ }^{1}$ \\ ${ }^{1}$ Department of Microbiology, University of Massachusetts, Amherst, MA, USA; ${ }^{2}$ Department of Physics, \\ University of Massachusetts, Amherst, MA, USA; ${ }^{3}$ Department of Physical and Biological Sciences, Western \\ New England University, Springfield, MA, USA and ${ }^{4}$ Department of Physics, Jacksonville University, \\ Jacksonville, FL, USA
}

\begin{abstract}
The possibility that bacteria other than Geobacter species might contain genes for electrically conductive pili (e-pili) was investigated by heterologously expressing pilin genes of interest in Geobacter sulfurreducens. Strains of $G$. sulfurreducens producing high current densities, which are only possible with e-pili, were obtained with pilin genes from Flexistipes sinusarabici, Calditerrivibrio nitroreducens and Desulfurivibrio alkaliphilus. The conductance of pili from these strains was comparable to native $G$. sulfurreducens e-pili. The e-pili derived from $C$. nitroreducens, and $D$. alkaliphilus pilin genes are the first examples of relatively long ( $>100$ amino acids) pilin monomers assembling into e-pili. The pilin gene from Candidatus Desulfofervidus auxilii did not yield e-pili, suggesting that the hypothesis that this sulfate reducer wires itself with e-pili to methane-oxidizing archaea to enable anaerobic methane oxidation should be reevaluated. A high density of aromatic amino acids and a lack of substantial aromatic-free gaps along the length of long pilins may be important characteristics leading to e-pili. This study demonstrates a simple method to screen pilin genes from difficult-to-culture microorganisms for their potential to yield e-pili; reveals new sources for biologically based electronic materials; and suggests that a wide phylogenetic diversity of microorganisms may use e-pili for extracellular electron exchange.
\end{abstract}

The ISME Journal (2018) 12, 48-58; doi:10.1038/ismej.2017.141; published online 5 September 2017

\section{Introduction}

Electrically conductive pili (e-pili) can have an important role in the biogeochemical cycling of carbon and metals and have potential applications as 'green' electronic materials (Lovley, 2011; Malvankar and Lovley, 2014; Lovley and Malvankar, 2015; Shi et al., 2016; Lovley, 2017a, $\mathrm{b}, \mathrm{c})$. Therefore, a better understanding of the diversity of microorganisms capable of producing e-pili has many potential benefits. To date, e-pili have been most intensively studied in Geobacter sulfurreducens and the closely related Geobacter metallireducens (Reguera et al., 2005; Malvankar et al., 2011, 2014, 2015; Vargas et al., 2013; Feliciano et al., 2015; Adhikari et al., 2016; LampaPastirk et al., 2016; Xiao et al., 2016; Tan et al., 2016b, 2017). These studies have demonstrated

Correspondence: DR Lovley, Department of Microbiology, University of Massachusetts, Morrill IV N Science Center, Amherst, MA 01003, USA.

E-mail: dlovley@microbio.umass.edu

${ }^{5}$ Current Address: Department of Physics, Jacksonville University, Jacksonville, FL, USA.

Received 17 March 2017; revised 17 June 2017; accepted 25 July 2017; published online 5 September 2017 that the e-pili of both $G$. sulfurreducens and G. metallireducens are sufficiently conductive along their length, under physiologically relevant conditions, to account for maximum potential rates of extracellular electron transfer (Adhikari et al., 2016; Lampa-Pastirk et al., 2016; Tan et al., 2016b, 2017). Geobacter strains that lack e-pili, or express pili with diminished conductivities, are incapable of direct interspecies electron transfer (DIET) and Fe(III) oxide reduction (Reguera et al., 2005; Summers et al., 2010; Tremblay et al., 2012; Shrestha et al., 2013; Vargas et al., 2013; Liu et al., 2014; Rotaru et al., 2014a, b; Tan et al., 2016b). e-Pili are also required for Geobacter species to generate high current densities when electrodes serve as the sole electron acceptor (Reguera et al., 2006; Vargas et al., 2013; Liu et al., 2014; Tan et al., 2016b). Increasing e-pili expression increases the conductivity of electrode biofilms and their current output (Yi et al., 2009; Malvankar et al., 2011, 2012; Leang et al., 2013).

Aromatic amino acids are key components in electron transfer along the length of $G$. sulfurreducens e-pili (Vargas et al., 2013; Adhikari et al., 2016; Lampa-Pastirk et al., 2016; Tan et al., 2016a, 2017). There is debate whether the aromatic amino acids contribute to a traditional electron-hopping electron 
transport mechanism or whether overlapping $\pi-\pi$ orbitals of the aromatics confer a metallic-like conductivity similar to that observed in synthetic conducting polymers (Malvankar et al., 2011, 2014, 2015; Vargas et al., 2013; Malvankar and Lovley, 2014; Lovley and Malvankar, 2015; Lampa-Pastirk et al., 2016). However, the uncertainty over these mechanistic details should not obscure the fact that long-range electron transport via e-pili is a remarkable strategy for long-range biological electron transfer.

It has been speculated that the relatively small size (61 amino acids) of the G. sulfurreducens pilin is a key feature contributing to its assembly into e-pili (Reguera et al., 2005; Malvankar et al., 2015; Holmes et al., 2016). Pili comprised of larger pilins, such as those found in Shewanella oneidensis (Reguera et al., 2005), Pseudomonas aeruginosa (Reguera et al., 2005; Liu et al., 2014) and G. uraniireducens (Tan et al., 2016b) have poor conductivity. On the basis of these results, it was proposed that smaller pilins might permit the tight packing of aromatic amino acids required for conductivity (Malvankar et al., 2015; Xiao et al., 2016). However, previously investigated poorly conductive pili comprised of longer pilins have either (1) a lower overall density of aromatic amino acids than those found in e-pili (that is, $P$. aeruginosa and S. oneidensis) or (2) a large gap within the pilin sequence devoid of aromatic amino acids, such as observed in the pilin of G. uraniireducens (Figure 1). These considerations suggest that the conductivity of a broader diversity of pili should be investigated.

Evaluating pili conductivity is technically challenging, especially with difficult-to-culture microorganisms. For example, it was suggested that e-pili were involved in DIET in an enriched consortium that contained a methane-oxidizing archaeon and its sulfate-reducing partner (Wegener et al., 2015). The sulfate-reducing partner highly expressed a pilin gene when the two microorganisms were growing syntrophically and pili-like filaments were abundant in the co-culture (Wegener et al., 2015). It was proposed that the pili of the sulfate reducer were conductive (Wegener et al., 2015), but this was not verified, possibly due to difficulties in obtaining sufficient biomass.

The properties of proteins encoded in genomes of difficult-to-culture or as-yet-uncultured microorganisms can often be determined by expressing the genes of interest in more readily cultivated microorganisms (Handelsman, 2004). It is possible to screen pilin genes to determine whether they have the potential to yield pilin proteins that can assemble into e-pili by heterologously expressing the pilin gene of interest in G. sulfurreducens in place of the native G. sulfurreducens pilin gene (Vargas et al., 2013; Liu et al., 2014; Tan et al., 2016a, b, 2017). Only if the pilin gene yields e-pili is G. sulfurreducens then capable of producing high current densities on graphite electrodes. For example, pili of $P$. aeruginosa are poorly conductive (Reguera et al., 2005) and a strain of $G$. sulfurreducens expressing the pilin gene of $P$. aeruginosa produced low current densities (Liu et al., 2014). Expression of the pilin gene from $G$. metallireducens in $G$. sulfurreducens yielded e-pili that were even more conductive than the e-pili originating from the native G. sulfurreducens pilin gene (Tan et al., 2017). In contrast, the pilin gene of $G$. uraniireducens, which is phylogenetically distinct from the $G$. sulfurreducens and G. metallireducens pilin genes (Holmes et al., 2016), yielded poorly conductive pili in G. sulfurreducens (Tan et al., 2016b). As expected, the G. sulfurreducens strain expressing the $G$. uraniireducens pilin genes produced low current densities (Tan et al., 2016b) as did a G. sulfurreducens strain expressing a synthetic pilin gene designed to yield poorly conductive pili (Vargas et al., 2013). The poor conductivity of the $G$. uraniireducens pili was consistent with the finding that $G$. uraniireducens uses an electron shuttle for Fe(III) reduction and is

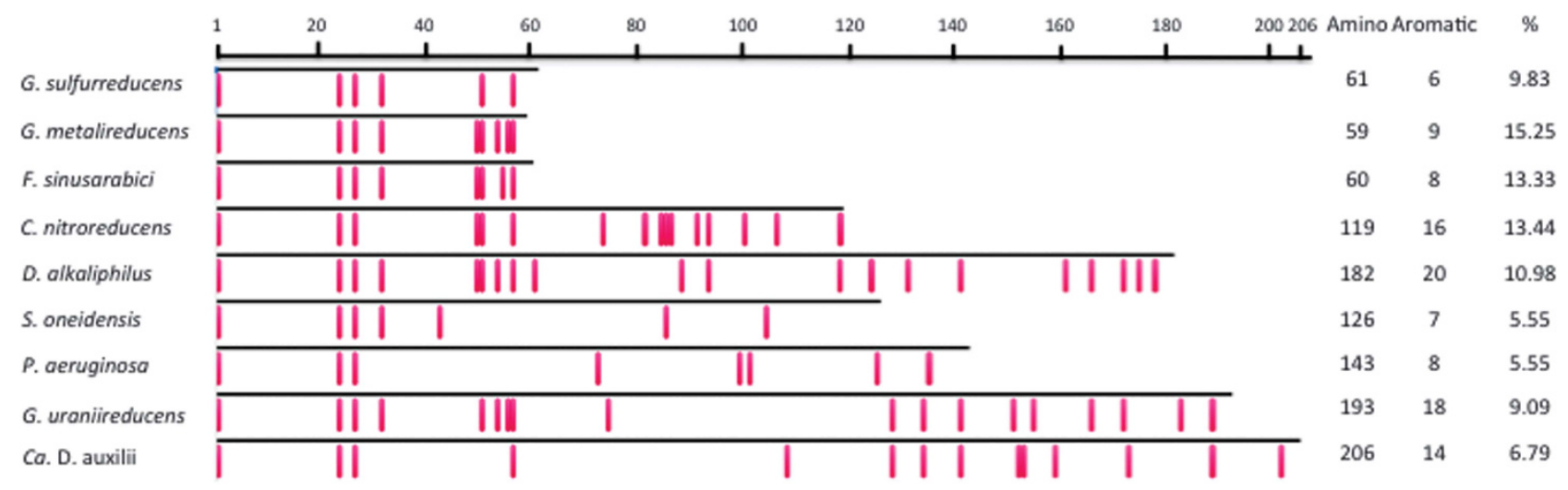

Figure 1 Comparison of aromatic amino-acid placement (red rectangles) within mature pilin protein sequences of G. sulfurreducens, G. metallireducens, F. sinusarabici, C. nitroreducens, D. alkaliphilus, S. oneidensis, P. aeruginosa, G. uraniireducens and Candidatus Desulfofervidus auxilii. Also shown are the total number of amino acids, the number of aromatic amino acids and the percentage of total amino acids that are aromatic. 
incapable of producing high current densities on graphite electrodes (Rotaru et al., 2015; Tan et al., 2016b).

Therefore, the possibility that diverse microorganisms potentially involved in extracellular electron transfer might have the potential to produce e-pili was evaluated by expressing their pilin genes in $G$. sulfurreducens. The results suggest that pilin sequences phylogenetically distinct from the $G$. sulfurreducens pilin and much larger than the $G$. sulfurreducens pilin can, in some instances, yield e-pili.

\section{Materials and methods}

Bacterial strains, plasmids and culture conditions The bacterial strains used in this study are listed in Supplementary Table S1. As previously described (Coppi et al., 2001), G. sulfurreducens was routinely cultured in liquid medium or on agar plates under strict anaerobic conditions in a medium with acetate as the electron donor and fumarate as the electron acceptor at $30^{\circ} \mathrm{C}$. Escherichia coli 5-alpha (New England Biolabs, Ipswich, MA, USA) chemically competent cells were used for Gibson Assembly cloning and cultured at $37^{\circ} \mathrm{C}$ in Luria-Bertani medium. Gentamicin $20 \mu \mathrm{g} \mathrm{ml}^{-1}$ was added to cultures when required for plasmid or chromosomal selection.

\section{Pilin gene sequences and phylogenetic analyses}

Sequence data were acquired from the US Department of Energy Joint Genome Institute (http://www. jgi.doe.gov) or from the Genbank at the National Center for Biotechnology Information (http://www. ncbi.nlm.nih.gov). Amino-acid alignments were generated with MAFFT (Katoh and Standleyu, 2013) and PRANK (Löytynoja and Goldman, 2005) algorithms, and unreliable residues, columns and sequences in all alignments were identified and eliminated with GUIDANCE2 (Sela et al., 2015).

Phylogenetic trees were generated with the maximum likelihood method using MEGA v. 7.0 software (Kumar et al., 2016). Before trees were constructed, the Find Best DNA/Protein Models program was run on sequence alignments. The evolutionary history of the PilA protein was inferred with the maximum likelihood method based on the Whelan and Goldman model (Whelan and Goldman, 2001) and the bootstrap consensus tree was inferred from 100 replicates (Felsenstein, 1985). Initial tree(s) for the heuristic search were obtained automatically by applying Neighbor-Joining and BioNJ algorithms to a matrix of pairwise distances estimated using a JTT model, and then selecting the topology with a superior log likelihood value. A discrete Gamma distribution was used to model evolutionary rate differences among sites (five categories $(+G$, parameter $=$ 0.9542)). The analysis involved 79 amino-acid sequences. All positions with $<95 \%$ site coverage were eliminated. That is, fewer than $5 \%$ alignment gaps, missing data and ambiguous bases were allowed at any position. There were a total of 55 positions in the final data set.

Construction of $G$. sulfurreducens strains expressing heterologous pilins

The strains of $G$. sulfurreducens heterologously expressing the pilin genes of other microorganisms were constructed with a slight modification of the previously described approach (Tan et al., 2017). Pilin genes (gene accession numbers in parentheses) were expressed from the following: Flexistipes sinusarabici DSM 4947 (Flexsi_2288); Calditerrivibrio nitroreducens DSM 19672 (Calni_0149); Desulfurivibrio alkaliphilus AHT2 (DaAHT2_2283) and Candidatus Desulfofervidus auxilii strain HS1 (HS1_000117). To ensure that the pre-pilin would be correctly recognized and transported to the cellular membrane for processing, the signal peptide from $G$. sulfurreducens (MLQKLRNRKG) was included in all strains. This signal peptide is cleaved during post-translational processing and is not part of the mature pilin sequence. The mature pilincoding sequence from the organisms of interest was inserted downstream from the $G$. sulfurreducens signal peptide sequence in the pYT-1 plasmid (Supplementary Figure S1). The region between the SacII and NotI restriction sites, which included the G. sulfurreducens promoter region (PpilA), the $G$. sulfurreducens signal peptide sequence and the heterologous pilA sequence, was synthesized as a gene fragment (Thermo Fisher Scientific, Waltham, MA, USA). Two 15-bp regions homologous to sequences located $5^{\prime}$ and $3^{\prime}$ of the restriction sites on the pYT-1 plasmid were also included in the synthesized gene fragment to facilitate Gibson Assembly. The synthesized DNA was inserted into plasmid pYT-1 between the SacII and NotI restriction sites with the Gibson Assembly Kit ((Gibson et al., 2009); New England Biolabs). Correct sequence and assembly was verified by sequencing DNA fragments amplified with pilA-Screen-F and pilAScreen-R primers (Supplementary Table S2). The recombinant plasmid was then linearized with restriction enzyme SacI and transformed into wild-type $G$. sulfurreducens competent cells by electroporation as previously described (Liu et al., 2014).

To ensure that the heterologous gene had correctly integrated into the G. sulfurreducens chromosome, sequences were verified with pilA-InScreen-F and pilA-InScreen-R primers (Supplementary Table S2). Strain purity was also confirmed by sequencing with this primer set throughout the experiment; that is, before placing the strain in the bioelectrochemical device, during the current production experiment and before pili were collected from cells. 


\section{Current production}

The capacity of the strains to produce current was determined as previously described (Nevin et al., 2009). Cells were grown in two-chambered H-cell systems with a continuous flow of medium with acetate $(10 \mathrm{~mm})$ as the electron donor and graphite stick anodes $\left(65 \mathrm{~cm}^{2}\right)$ poised at $300 \mathrm{mV}$ versus $\mathrm{Ag}$ / $\mathrm{AgCl}$ as the electron acceptor.

\section{Microscopy}

As previously described (Nevin et al., 2011), anode biofilms were imaged with confocal laser scanning microscopy using the LIVE/DEAD BacLight viability stain kit (Molecular Probes, Eugene, OR, USA). Cells from the anode biofilms were examined by transmission electron microscopy as previously described (Tan et al., 2017). Cells placed on carbon-coated copper grids were stained with $2 \%$ uranyl acetate and visualized with a JEOL (Welwyn garden city, UK) 2000fx transmission electron microscope operated at $200 \mathrm{kV}$ accelerating voltage.

\section{Pili preparation}

Pili were obtained from anode biofilms as previously described (Tan et al., 2017). Briefly, pili were sheared from the cells in a blender in $150 \mathrm{~mm}$ ethanolamine buffer ( $\mathrm{pH}$ 10.5), the cells were removed with centrifugation and the pili were precipitated from the supernatant with the addition of ammonium sulfate, followed by centrifugation. The precipitate was resuspended in ethanolamine buffer, centrifuged to remove additional debris and the ammonium sulfate precipitation step was repeated. The pili were then resuspended in ethanolamine buffer and stored at $4{ }^{\circ} \mathrm{C}$. Before conductance measurements the pili were dialyzed in deionized water at $4{ }^{\circ} \mathrm{C}$ with a Slide-A-Lyzer MINI Dialysis Device (Thermo Fisher Scientific) with $7 \mathrm{kDa}$ molecular weight cutoff columns. The purity of the pili preparations was evaluated with transmission electron microscopy, as previously described (Tan et al., 2016a). To determine the protein concentration, $20 \mu \mathrm{l}$ of the pili preparation was air dried, dissociated in $17.2 \mu \mathrm{l}$ of $1 \%$ SDS at $\mathrm{pH} 1.5$ at $95^{\circ} \mathrm{C}$ and then neutralized with $2.8 \mu \mathrm{l}$ of $1 \mathrm{~N} \mathrm{NaOH}$. The protein concentration was analyzed with the Pierce nano bicinchoninic acid assay (Thermo Fisher) All pili preparations were normalized to $500 \mu \mathrm{g}$ protein per $\mu \mathrm{l}$.

\section{Pili conductance}

The method for measuring pili conductance was a modification of the previously described (Malvankar et al., 2011; Vargas et al., 2013) method for estimating the conductance of pili networks. This method is preferable for screening studies because measuring the conductivity of multiple individual pili is extremely laborious, expensive and technically difficult. Conductance was measured with the four-point probe method to eliminate the possibility of contact resistance.

The electrodes were fabricated with photolithography and were $50 \mathrm{~nm}$ thick with $10 \mathrm{~nm}$ titanium used as an adhesion layer and $40 \mathrm{~nm}$ gold (Supplementary Figure S2). The substrate was $300 \mathrm{~nm}$-thick silicon dioxide grown on antimonydoped silicon wafers with a wet chemical process. Pads for the four-point probe electrodes were $1 \mathrm{~mm}$ by $1 \mathrm{~mm}$ in length and the electrodes were nonequidistant with $15 \mu \mathrm{m}$ between the two inner electrodes and $3 \mu \mathrm{m}$ between each of the inner and outer electrodes. Electrical contacts on the pads were made with $1 \mu \mathrm{m}$ diameter tungsten probes (Signatone, Gilroy, CA, USA) connected to a Keithley 4200 Semiconductor Characterization System Parametric Analyzer (Tektronix Inc., Beaverton, OR, USA) with triaxial cables.

Pili preparations $(2 \mu \mathrm{l}, 500 \mu \mathrm{g}$ protein per $\mu \mathrm{l})$ were drop cast onto the gold electrode arrays and dried for $1 \mathrm{~h}$ at $24^{\circ} \mathrm{C}$. Another $2 \mu \mathrm{l}$ of the pili preparation was then drop cast on the electrode array and dried in a desiccator for ca. $12 \mathrm{~h}$.

Each pili preparation was drop cast onto three different devices and current-voltage $(I-V)$ curves for each device were obtained in triplicate with a Keithley 4200 Semiconductor Characterization System setup with four probes using a $\pm 30 \times 10^{-8} \mathrm{~V}$ sweep with a $5 \mathrm{~s}$ delay and a $250 \mathrm{~s}$ hold time. The conductance value of the pili was extracted from the slope of the linear fit of the current-voltage response of the sample as

$G=\frac{I}{V}$

where $G$ is the conductance, $I$ is the current and $V$ is the voltage.

\section{Results and discussion}

e-Pili from the Geobacter-like pilin of F. sinusarabici Many Geobacter species and closely related microorganisms have pilin genes that are closely related to the G. sulfurreducens pilin gene that yields e-pili (Holmes et al., 2016). These include other members of the Desulfuromonadales family in the Deltaproteobacteria, such as some Geoalkalibacter, Desulfuromusa and Desulfuromonas species that are already known to participate in extracellular electron transfer (Holmes et al., 2016). However, a few species outside the Desulfuromonadales also have pilin genes closely related to those found in G. sulfurreducens (Holmes et al., 2016). For example, $F$. sinusarabici, which is in the phylum Deferribacteres, is phylogenetically distant from Geobacter species based on 16S rRNA gene sequences, but contains a pilin gene closely related to the $G$. sulfurreducens pilin gene (Holmes et al., 2016) that is predicted to yield a pilin monomer with a density 
of aromatic amino-acid intermediate between the $G$. sulfurreducens and $G$. metallireducens pilins (Figure 1).

Even under optimal culture conditions $F$. sinusarabici grows poorly, not even producing visible turbidity (Fiala et al., 1990). Thus, it was not possible to obtain sufficient biomass to evaluate the conductivity of $F$. sinusarabici pili directly. Therefore, the pilin gene of $F$. sinusarabici was heterologously expressed in G. sulfurreducens in place of the native $G$. sulfurreducens pilin gene. The $G$. sulfurreducens strain expressing the $F$. sinusarabici pilin gene grew on anodes, and produced current densities comparable to wild-type G. sulfurreducens (Figure 2). Such high current densities have only been seen in previous studies when G. sulfurreducens expresses e-pili (Reguera et al., 2006; Vargas et al., 2013; Liu et al., 2014; Tan et al., 2016b, 2017), which permit cells at distance from the anode to contribute to current production (Lovley, 2012). The thick biofilms of the $G$. sulfurreducens strain expressing the $F$. sinusarabici pilin gene (Figure 3a) were comparable to those previously reported (Vargas et al., 2013) for the control strain expressing the G. sulfurreducens wild-type pilin gene.

The G. sulfurreducens strain expressing the $F$. sinusarabici pilin gene expressed abundant pili (Figure 4a). The purified pili (Supplementary Figure S3) had a conductance comparable to that of the pili preparations from the control strain of $G$. sulfurreducens expressing its native pilin gene (Figure 5). These results demonstrate that the $F$. sinusarabici pilin gene encodes a pilin monomer that can assemble into e-pili. Close relatives of F. sinusarabici include Geovibrio and Deferribacter species, which are known to be capable of extracellular electron transfer (Alauzet and Jumas-Bilak, 2014). The one available Deferribacter genome contains a pilin gene homologous to the $G$. sulfurreducens pilin gene (Holmes et al., 2016). Further investigation into the possibility that $F$. sinusarabici uses e-pili for extracellular electron transfer is warranted, but such studies will be challenging because of its poor growth and the lack of tools for genetic manipulation.

e-Pili from larger, more phylogenetic distant pilins The possibility that pilins that are larger than the truncated e-pilin of $G$. sulfurreducens might also yield e-pili has only been previously explored with a few pilin sequences (Liu et al., 2014; Tan et al., 2016b). Therefore, larger pilin sequences were further investigated with an emphasis on microorganisms that (1) are thought to be capable of extracellular electron exchange and (2) have aromatic-rich pilin proteins.

For example, C. nitroreducens, which like $F$. sinusarabici is in the phylum Deferribacteres, is capable of extracellular electron transfer to electrodes (Fu et al., 2013), and the density of aromatic

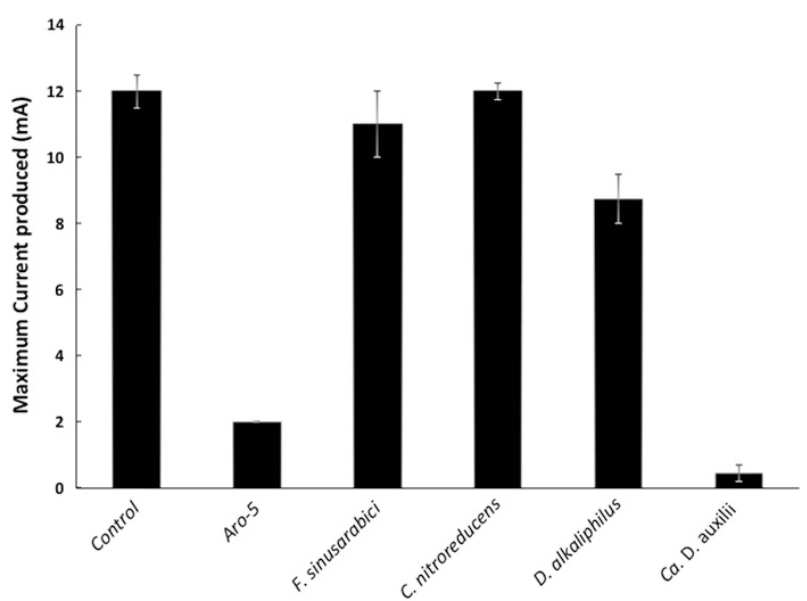

Figure 2 Current production by G. sulfurreducens strains expressing either the native wild-type gene (control), a synthetic pilin gene (Aro-5) designed to be poorly conductive (Vargas et al., 2013 ) or the pilin gene of $F$. sinusarabici, $C$. nitroreducens, D. alkaliphilus or Ca. D. auxilii. Results shown are the mean of duplicate determinations for each strain with the bars designating the two current values. Aro-5 results from Vargas et al. (2013).

amino acids in the $C$. nitroreducens pilin protein is higher than in the G. sulfurreducens pilin (Figure 1). However, the $C$. nitroreducens pilin contains nearly twice as many amino acids as the G. sulfurreducens pilin. A strain of $G$. sulfurreducens expressing the $C$. nitroreducens pilin gene, rather than the native G. sulfurreducens pilin gene, produced current densities similar to the control strain expressing the native pilin gene (Figure 2) with thick biofilms (Figure 3b). The cells produced abundant pili (Figure 4b). The conductance of the purified pili preparations (Supplementary Figure S3) from the anode biofilm was almost twice as that of the control strain expressing the native G. sulfurreducens pilin gene (Figure 5). These results demonstrate for the first time that pilin monomers much larger than the pilin of G. sulfurreducens can yield e-pili.

Further investigation of the role of e-pili in C. nitroreducens will be challenging because of the lack of tools for genetic manipulation of this organism and because this microorganism is difficult to grow to high densities. However, the possibility of C. nitroreducens expressing e-pili should be considered when evaluating its ecological niche or possibilities for increasing its current output in microbial fuel cells.

It has been proposed that cable bacteria accept electrons from sulfide-oxidizing bacteria via DIET (Vasquez-Cardenas et al., 2015). Genome sequences of cable bacteria have yet to be published but a genome is available for D. alkaliphilus (Melton et al., 2016), which is closely related to some species of cable bacteria (Müller et al., 2016). Similar to Geobacter species, D. alkaliphilus is in the Deltaproteobacteria. However, the pilin of $D$. alkaliphilus is more closely related to the pilin of $G$. uraniireducens, which yields poorly conductive pili (Tan et al., 2016b), than to the G. sulfurreducens pilin (Figure 6). 

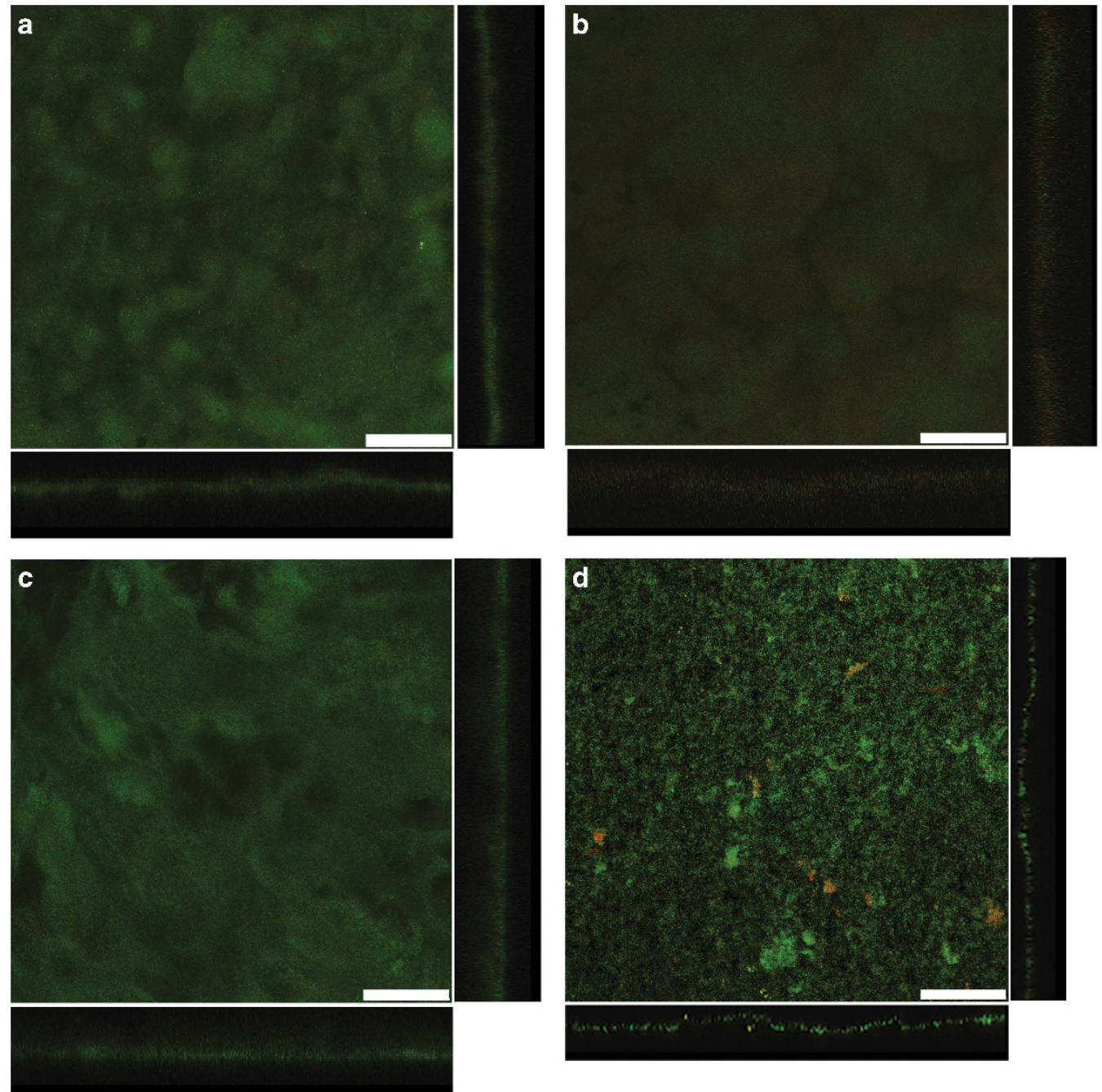

Figure 3 Scanning laser confocal microscopy images of anode biofilms of $G$. sulfurreducens strains heterologously expressing pilin genes from (a) F. sinusarabici, (b) C. nitroreducens, (d) D. alkaliphilus and (d) Ca. D. auxilii. Top-down three-dimensional, lateral side views (right image) and horizontal side views (bottom image) show cells stained with LIVE/DEAD BacLight viability stain. Bar, $50 \mu m$. Mean and s.d. for biofilm thickness at 100 points in each biofilm: F. sinusarabici, $26.1 \pm 5.2 \mu \mathrm{m}$; C. nitroreducens, $41.0 \pm 6.6 \mu \mathrm{m}$; D. alkaliphilus, $41.7 \pm 6.6 \mu \mathrm{m}$; Ca. D. auxilii, $10.7 \pm 2.1 \mu \mathrm{m}$.

Similar to the G. uraniireducens pilin, the D. alkaliphilus pilin is much larger than the pilins that have been shown to yield e-pili in Geobacter species (Figure 1). However, unlike the G. uraniireducens pilin, the density of aromatic amino acids in the $D$. alkaliphilus pilin is comparable to that of $G$. sulfurreducens (Figure 1). Expression of the $D$. alkaliphilus pilin gene in $G$. sulfurreducens yielded a strain that produced thick anode biofilms (Figure 3c) that generated high current densities (Figure 2). Pili were abundant (Figure 4c). The purified pili preparations (Supplementary Figure S3) had a conductance nearly equal to native $G$. sulfurreducens pili (Figure 5). These results provide another example of a large pilin monomer yielding e-pili.

It does not appear that the ability of $D$. alkaliphilus to reduce Fe(III) oxides or produce current has been examined, but $D$. alkaliphilus does contain porincytochrome genes closely related to Geobacter genes that are known to be involved in extracellular electron transfer (Shi et al., 2014). Furthermore, D. alkaliphilus grows with elemental sulfur/polysulfide as the electron acceptor (Sorokin et al., 2008) and microorganisms that can reduce elemental sulfur/ polysulfide are often also capable of electron transfer to Fe(III) or other extracellular electron acceptors (Lovley et al., 2004). If cable bacteria closely related to $D$. alkaliphilus have similar pilin genes, this might provide a mechanism for the proposed DIET between cable bacteria and sulfur oxidizers (VasquezCardenas et al., 2015). The fact that cable bacteria or their putative sulfur-oxidizing partners are not available in pure culture currently limits further investigation into this possibility.

Pilin of Ca. D. auxilii does not yield e-pili

It has been proposed that the sulfate-reducer $C a$. D. auxilii has e-pili that facilitate DIET in a 
consortium with a methane-oxidizing archaeon (Wegener et al., 2015; Krukenberg et al., 2016). The phylogeny of $C a$. D. auxilli is still under evaluation, but it is either a member of the Deltaproteobacteria or the closely related Thermodesulfobacteria (Krukenberg et al., 2016). It was not possible for us to obtain a sample of the consortium to directly evaluate the conductivity of the pili, but $G$. sulfurreducens readily
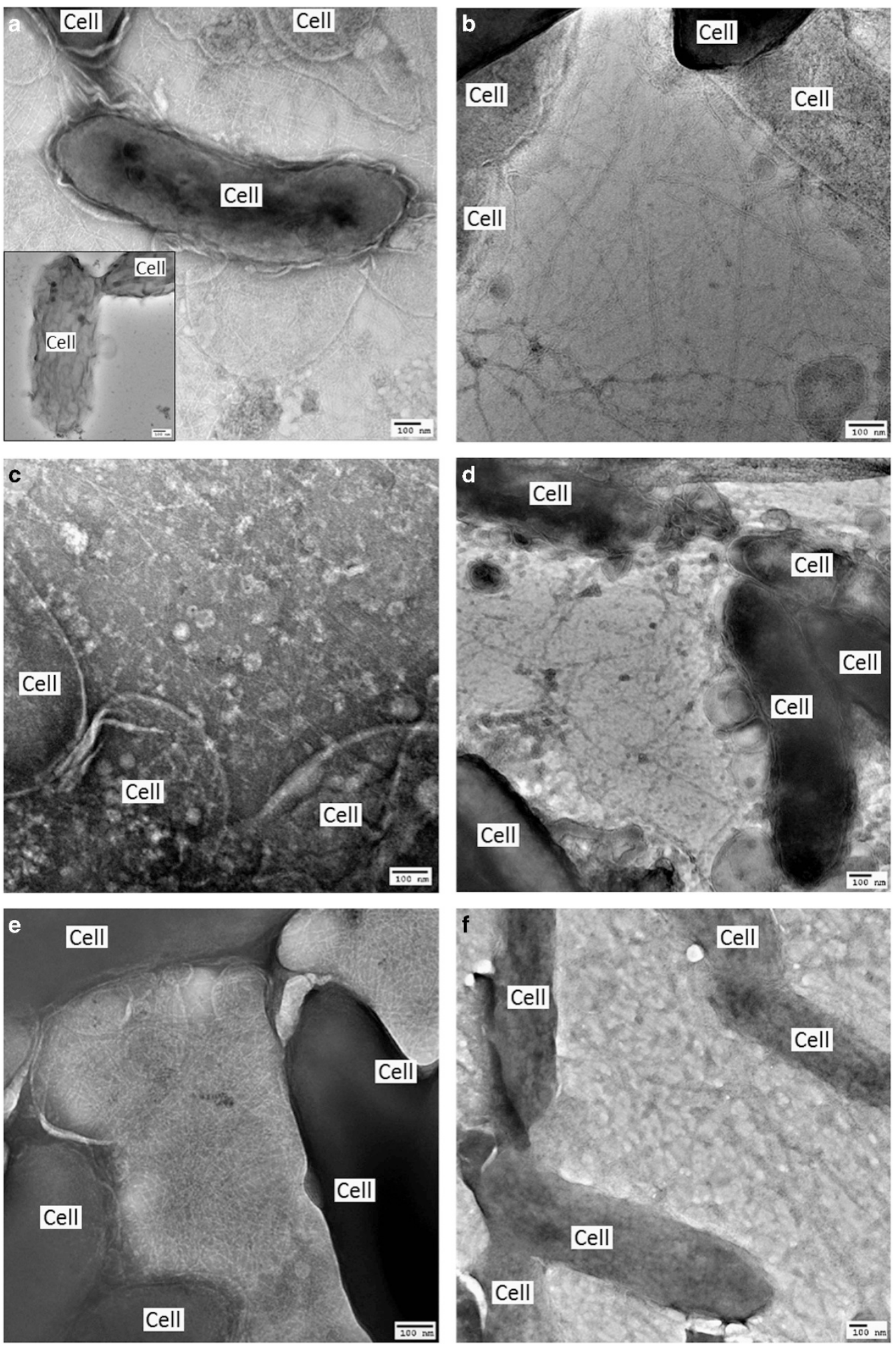

Figure 4 Transmission electron micrographs of G. sulfurreducens strains. (a) Control strain expressing wild-type pilin gene (insert: strain with pilin gene deleted). Heterologous expression of pilin genes from the following organisms: (b) F. sinusarabici, (c) C. nitroreducens, (d) D. alkaliphilus, (e) Ca. D. auxilii or (f) the synthetic Aro-5 sequence. 


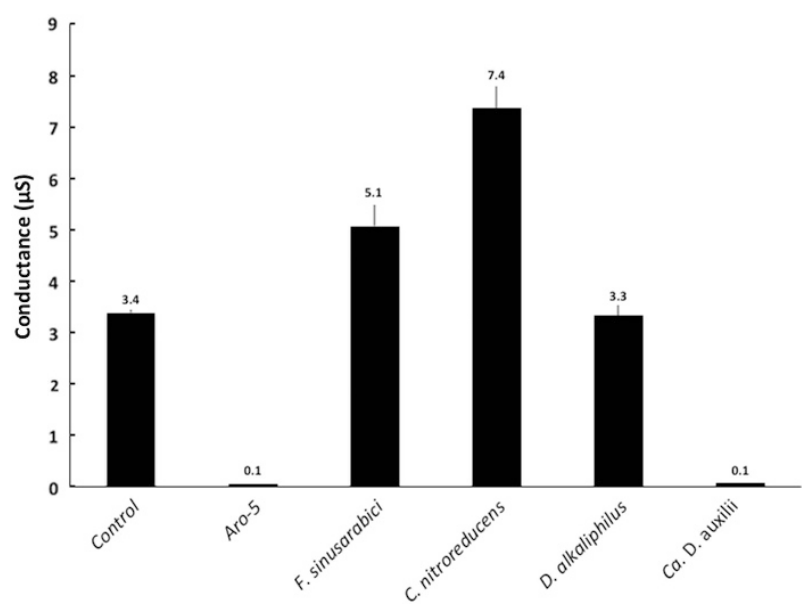

Figure 5 Conductance of pili preparations from G. sulfurreducens strains expressing either the native wild-type gene (control), a synthetic pilin gene (Aro-5) designed to be poorly conductive (Vargas et al., 2013) or the pilin gene of $F$. sinusarabici, C. nitroreducens, $D$. alkaliphilus or $C a$. D. auxilii. Results shown are the mean and s.d. of triplicate measurements on triplicate electrode arrays.

expressed pili (Figure 4d; Supplementary Figure S3) from the $\mathrm{Ca}$. D. auxilli pilin gene that was previously reported (Wegener et al., 2015) to be highly expressed during syntrophic growth. This strain of G. sulfurreducens produced low current densities (Figure 2) with relatively thin biofilms (Figure 3d), comparable to the previously reported (Liu et al., 2014) biofilms produced by a strain of $G$. sulfurreducens expressing the pilin from $P$. aeruginosa, which yield poorly conductive pili. The conductance of the pili was also low (Figure 5). There is the possibility that the $\mathrm{Ca}$. D. auxilli pilin monomers assemble differently in $C a$. D. auxilli than they do in G. sulfurreducens to yield conductive pili in the natural host. However, this seems unlikely due to the finding that $G$. sulfurreducens expressed e-pili from the pilin monomer of $D$. alkaliphilus, which is comparable in size. The density of aromatic amino acids in the Ca. D. auxilli pilin is much lower than that found in e-pili (Figure 1), suggesting that even when expressed in the native host the pilin of $\mathrm{Ca}$. D. auxilli is unlikely to assemble into a conductive filament. These results suggest that the hypothesis that $\mathrm{Ca}$. D. auxilli exchanges electrons with its archeal partner via $C a$. D. auxilli e-pili (Wegener et al., 2015) needs to be revisited. One possible approach would be to measure conductance of the consortium as such measurements can be more technically feasible than studying pili conductance directly (Morita et al., 2011; Shrestha et al., 2014).

\section{Conclusions}

These results demonstrate that microorganisms outside the Geobacteraceae contain pilin genes that can encode e-pili and that pilin monomers much larger than those of $G$. sulfurreducens can yield e-pili. Thus, the diversity of microorganisms that might electrically communicate with other species, or exchange electrons with insoluble minerals, through e-pili may extend well beyond the small percentage of microbes with genes for short e-pilins similar to those found in G. sulfurreducens.

The poor understanding of the mechanisms for conductivity for even $G$. sulfurreducens e-pili precludes definitively specifying the features of pilin monomers that confer conductivity to e-pili. However, the limited data set available suggests that pilins with high densities of aromatic amino acids, without large gaps of aromatic-free regions in the pilin monomer, can assemble into e-pili. The phylogenetic distance between the longer pilin sequences found to yield e-pili and the shorter pilin sequences closely related to $G$. sulfurreducens (Figure 6) suggest that aromatic-rich e-pili may have independently evolved several times within bacteria.

The results offer a simple screening strategy to evaluate whether difficult-to-culture microorganisms contain pilin genes that might enable them to participate in extracellular electron exchange with e-pili. The genetic manipulation of $G$. sulfurreducens to heterologously express pilin genes of interest is straightforward and the requirement of $G$. sulfurreducens for e-pili to produce high current densities provides a clear phenotype. Thus, the approach described here provides a good first step for hypothesis testing and is preferable to the common practice of suggesting that filaments are 'nanowires' based solely on visual appearance. Ideally, detailed investigation of the function of the e-pili from any microorganism should also include estimates of the conductivity of individual e-pili under physiologically relevant conditions (Adhikari et al., 2016; Tan et al., 2016a, b, 2017). However, such measurements are technically challenging and the necessary equipment and expertise are not readily available to many microbiologists.

Diverse microorganisms such as Aeromonas hydrophila (Castro et al., 2013), Acidithiobacillus ferroxidans (Li and Li, 2014), Desulfovibrio desulfuricans (Eaktasang et al., 2016) and Rhodopseudomonas palustris (Venkidusamy et al., 2015) can produce electrically conductive protein filaments. However, uncertainties about the physiological roles of these filaments exist because of the following: (1) the protein composition of the filaments has not been determined; (2) a role for the filaments in extracellular electron transfer has not been verified; and (3) electron transport along the length of filaments under physiologically relevant conditions has yet to be shown. Expressing pilin genes from these microbes in G. sulfurreducens may help better define these filaments.

e-Pili are a potential source of 'green' electronic materials that can be sustainably mass-produced with renewable feedstocks without toxic components in the final product (Lovley, 2017b). The e-pili revealed here greatly expand the options for starting materials and the screening method described will 


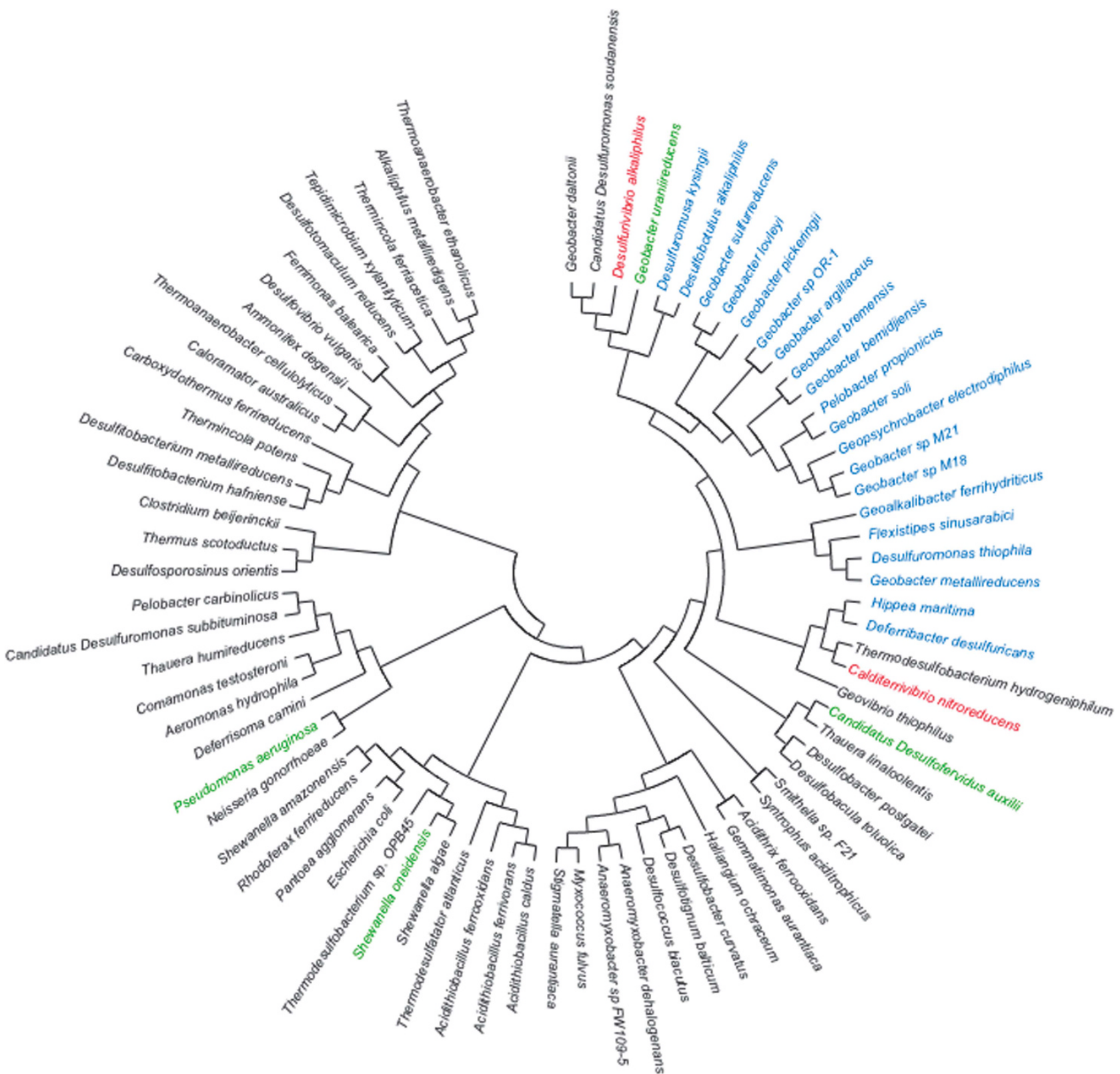

Figure 6 Phylogeny of pilin protein sequences from a diversity of bacteria.Short (ca. 60 amino acids) pilins closely related to $G$. sulfurreducens are shown in blue, including the pilin from $F$. sinusarabici (bold). The phylogenetic placement of the longer pilins from C. nitroreducens and D. alkaliphilus that assembled into conductive pili in G. sulfurreducens are in red. The pilins from Ca. D. auxilii, $S$. oneidensis and $P$. aeruginosa that did not yield conductive pili in this or previous studies are shown in green.

facilitate searching the microbial world, including the metagenomes of uncultured microorganisms, for additional conductive protein wires.

\section{Conflict of Interest}

The authors declare no conflict of interest.

\section{Acknowledgements}

This research was supported by Office of Naval Research Grants N000141310549 and N000141612526.

\section{References}

Adhikari RY, Malvankar NS, Tuominen MT, Lovley DR. (2016). Conductivity of individual Geobacter pili. RSC Adv 6: 8354-8357.

Alauzet C, Jumas-Bilak E. (2014). The phylum Deferribacteres and the genus Caldithrix. In: al. ERe (ed.). The Prokaryotes-Other Major Lineages of Bacteria and the Archaea. Springer-Verlag: Berlin, Germany, pp 595-611.

Castro L, Vera M, Muñoz JA, Blázquez ML, González F, Sand W et al. (2013). Conductive filaments produced by Aeromonas hydrophila. Adv Mater Res $\mathbf{8 2 5}$ : 210-213. 
Coppi MV, Leang C, Sandler SJ, Lovley DR. (2001). Development of a genetic system for Geobacter sulfurreducens. Appl Environ Microbiol 67: 3180-3187.

Eaktasang N, Kang CS, Lim H, Kwean OS, Cho S, Kim Y et al. (2016). Production of electrically-conductive nanoscale filaments by sulfate- reducing bacteria in the microbial fuel cell. Bioresour Technol 201: 61-67.

Feliciano GT, Steidl RJ, Reguera G. (2015). Structural and functional insights into the conductive pili of Geobacter sulfurreducens revealed in molecular dynamics simulations. Phys Chem Chem Phys 17: 22217-22226.

Felsenstein J. (1985). Confidence limits on phylogenies: an approach using the bootstrap. Evolution 39: 783-791.

Fiala G, Woese CR, Langworthy TA, Stetter KO. (1990). Flexistipes sinusarabici, a novel genus and species of eubacteria occurring in the Atlantis II deep brines of the Red Sea. Arch Microbiol 154: 120-126.

Fu Q, Kobayashi H, Kawaguchi H, Wakayama T, Maeda H, Sato K. (2013). A thermophilic gram-negative nitratereducing bacterium, Calditerrivibrio nitroreducens, exhibiting electricity generation capability. Environ Sci Technol 47: 12583-12590.

Gibson DG, Young L, Chuang RY, Venter JC, Hutchison CA, Smith HO. (2009). Enzymatic assembly of DNA molecules up to several hundred kilobases. Nat Methods 6: 343-345.

Handelsman J. (2004). Metagenomics: application of genomics to uncultured microorganisms. MIcrobiol Mol Biol Rev 68: 669-685.

Holmes DE, Dang Y, Walker DJF, Lovley DR. (2016). The electrically conductive pili of Geobacter species are a recently evolved feature for extracellular electron transfer. Microb Genom 2: e000072.

Katoh K, Standleyu DM. (2013). MAFFT multiple sequence alignment software version 7: improvements in performance and usability. Mol Biol Evol 30: 772-780.

Krukenberg V, Harding K, Richter M, Glockner FO, Gruber-Vodicka HR, Adam B et al. (2016). Candidatus Desulfofervidus auxilii, a hydrogenotrophic sulfatereducing bacterium involved in the thermophilic anaerobic oxidation of methane. Environ Microbiol 18: 3073-3091.

Kumar S, Stecher G, Tamura K. (2016). MEGA7: Molecular Evolutionary Genetics Analysis version 7.0 for bigger datasets. Mol Biol Evol 33: 1870-1874.

Lampa-Pastirk S, Veazey JP, Walsh KA, Feliciano GT, Steidl RJ, Tessmer S et al. (2016). Thermally activated charge transport in microbial protein nanowires. Sci Rep 6: 23517.

Leang C, Malvankar NS, Franks AE, Nevin KP, Lovley DR. (2013). Engineering Geobacter sulfurreducens to produce a highly cohesive conductive matrix with enhanced capacity for current production. Energy Environ Sci 6: 1901-1908.

Li Y, Li H. (2014). Type IV pili of Acidithiobacillus ferrooxidans can transfer electrons from extracellular electron donors. J Basic Microbiol 54: 226-231.

Liu X, Tremblay P-L, Malvankar NS, Nevin KP, Lovley DR, Vargas M. (2014). A Geobacter sulfurreducens strain expressing Pseudomonas aeruginosa type IV pili localizes OmcS on pili but Is deficient in Fe(III) oxide reduction and current production. Appl Environ Microbiol 80: 1219-1224.

Lovley DR, Holmes DE, Nevin KP. (2004). Dissimilatory Fe (III) and Mn(IV) reduction. Adv Microb Physiol 49: 219-286.
Lovley DR. (2011). Live wires: direct extracellular electron exchange for bioenergy and the bioremediation of energy-related contamination. Energy Environ Sci 4: 4896-4906.

Lovley DR. (2012). Electromicrobiology. Ann Rev Microbol 66: 391-409.

Lovley DR, Malvankar NS. (2015). Seeing is believing: novel imaging techniques help clarify microbial nanowire structure and function. Environ Microbiol 7: 2209-2215.

Lovley DR. (2017a). Happy together: microbial communities that hook up to swap electrons. ISME $J$ 11: $327-336$.

Lovley DR. (2017b). e-Biologics: fabrication of sustainable electronics with 'green' biological materials. mBio 8: e00695-17.

Lovley DR. (2017c). Syntrophy goes electric: direct interspecies electron transfer (DIET). Ann Rev Microbiol 71: 643-664.

Löytynoja A, Goldman N. (2005). An algorithm for progressive multiple alignment of sequences with insertions. Proc Natl Acad Sci USA 102: 10557-10562.

Malvankar NS, Vargas M, Nevin KP, Franks AE, Leang C, Kim B-C et al. (2011). Tunable metallic-like conductivity in nanostructured biofilms comprised of microbial nanowires. Nat Nanotechnol 6: 573-579.

Malvankar NS, Tuominen MT, Lovley DR. (2012). Biofilm conductivity is a decisive variable for high-currentdensity Geobacter sulfurreducens microbial fuel cells. Energy Environ Sci 5: 5790-5797.

Malvankar NS, Lovley DR. (2014). Microbial nanowires for bioenergy applications. Curr Opin Biotechnol 27: 88-95.

Malvankar NS, Yalcin SE, Tuominen MT, Lovley DR. (2014). Visualization of charge propagation along individual pili proteins using ambient electrostatic force microscopy. Nat Nanotechnol 9: 1012-1017.

Malvankar NS, Vargas M, Nevin KP, Tremblay P-L, EvansLutterodt K, Nykypanchuk D et al. (2015). Structural basis for metallic-like conductivity in microbial nanowires. mBio 6: e00084-00015.

Melton ED, Sorokin DY, Overmars L, Chertkov O, Clum A, Pillay M et al. (2016). Complete genome sequence of Desulfurivibrio alkaliphilus strain AHT2T, a haloalkaliphilic sulfidogen from Egyptian hypersaline alkaline lakes. Stand Genomic Sci 11: 67.

Morita M, Malvankar NS, Franks AE, Summers ZM, Giloteaux L, Rotaru AE et al. (2011). Potential for direct interspecies electron transfer in methanogenic wastewater digester aggregates. mBio 2: e00159-00111.

Müller H, Bosch J, Gribler C, Damgaard LR, Nielsen LP, Lueders T et al. (2016). Long-distance electron transfer by cable bacteria in aquifer sediments. ISME $J \mathbf{1 0}$ : 2010-2019.

Nevin KP, Kim B-C, Glaven RH, Johnson JP, Woodard TL, Methé BA et al. (2009). Anode biofilm transcriptomics reveals outer surface components essential for high current power production in Geobacter sulfurreducens fuel cells. PLoS One 4: e5628.

Nevin KP, Hensley SA, Franks AE, Summers ZM, Ou J, Woodard TL et al. (2011). Electrosynthesis of organic compounds from carbon dioxide is catalyzed by a diversity of acetogenic microorganisms. Appl Environ Microbiol 77: 2882-2886. 
Reguera G, McCarthy KD, Mehta T, Nicoll JS, Tuominen MT, Lovley DR. (2005). Extracellular electron transfer via microbial nanowires. Nature 435: 1098-1101.

Reguera G, Nevin KP, Nicoll JS, Covalla SF, Woodard TL, Lovley DR. (2006). Biofilm and nanowire production leads to increased current in Geobacter sulfurreducens fuel cells. Appl Environ Microbiol 72: 7345-7348.

Rotaru A-E, Shrestha PM, Liu F, Nevin KP, Lovley DR. (2014a). Direct interspecies electron transfer during syntrophic growth of Geobacter metallireducens and Methanosarcina barkeri on ethanol. Appl Environ Microbiol 80: 4599-4605.

Rotaru A-E, Shrestha PM, Liu F, Shrestha M, Shrestha D, Embree $\mathrm{M}$ et al. (2014b). A new model for electron flow during anaerobic digestion: direct interspecies electron transfer to Methanosaeta for the reduction of carbon dioxide to methane. Energy Environ Sci 7: 408-415.

Rotaru A-E, Woodard TL, Nevin KP, Lovley DR. (2015). Link between capacity for current production and syntrophic growth in Geobacter species. Front Microbiol 6: 744 .

Sela I, Ashkenazy H, Katoh K, Pupko T. (2015). GUIDANCE2: accurate detection of unreliable alignment regions accounting for theuncertainty of multiple parameters. Nucleic Acids Res 43: W7-W14.

Shi L, Fredrickson JK, Zachara JM. (2014). Genomic analysis of bacterial porin-cytochrome gene clusters. Front Microbiol 5: 657.

Shi L, Dong H, Reguera G, Beyenal H, Lu A, Liu J et al. (2016). Extracellular electron transfer mechanisms between microorganisms and minerals. Nat Rev Microbiol 14: 651-662.

Shrestha PM, Rotaru A-E, Summers ZM, Shrestha M, Liu F, Lovley DR. (2013). Transcriptomic and genetic analysis of direct interspecies electron transfer. Appl Environ Microbiol 79: 2397-2404.

Shrestha PM, Malvankar NS, Werner JJ, Franks AE, Rotaru A-E, Shrestha M et al. (2014). Correlation between microbial community and granule conductivity in anaerobic bioreactors for brewery wastewater treatment. Bioresource Tech 174: 306-310.

Sorokin DY, Tourova TP, Mufmann M, Muyzer G. (2008). Dethiobacter alkaliphilus gen. nov. sp. nov., and Desulfurivibrio alkaliphilus gen. nov. sp. nov.: two novel representatives of reductive sulfur cycle from soda lakes. Extremophiles 12: 431-439.

Summers ZM, Fogarty H, Leang C, Franks AE, Malvankar NS, Lovley DR. (2010). Direct exchange of electrons within aggregates of an evolved syntrophic co-culture of anaerobic bacteria. Science 330: 1413-1415.
Tan Y, Adhikari RY, Malvankar NS, Pi S, Ward JE, Woodard TL et al. (2016a). Synthetic biological protein nanowires with high conductivity. Small 12: 4481-4485.

Tan Y, Adhikari RY, Malvankar NS, Ward JE, Nevin KP, Woodard TL et al. (2016b). The low conductivity of Geobacter uraniireducens pili suggests a diversity of extracellular electron transfer mechanisms in the genus Geobacter. Front Microbiol 7: 980.

Tan Y, Adhikari RY, Malvankar NS, Ward JE, Woodard TL, Nevin KP et al. (2017). Expressing the Geobacter metallireducens PilA in Geobacter sulfurreducens yields pili with exceptional conductivity. mBio 8: e02203-e02216.

Tremblay P-L, Aklujkar M, Leang C, Lovley DR. (2012). A genetic system for Geobacter metallireducens: role of flagella and pili in extracellular electron transfer. Environ Microbiol Rep 4: 82-88.

Vargas M, Malvankar NS, Tremblay P-L, Leang C, Smith JA, Patel P et al. (2013). Aromatic amino acids required for pili conductivity and long-range extracellular electron transport in Geobacter sulfurreducens. mBio 4: e00105-e00113.

Vasquez-Cardenas D, van de Vossenberg J, Polerecky L, Malkin SY, Schauer R, Hidalgo-Martinez S et al. (2015). Microbial carbon metabolism associated with electrogenic sulphur oxidation in coastal sediments. ISME J 9: 1966-1978.

Venkidusamy K, Megharah M, Schroder U, Karouta F, Mohan SV, Naidu R. (2015). Electron transport through electrically conductive nanofilaments in Rhodopseudomonas palustris strain RP2. RSC Adv 5: 100790-100798.

Wegener G, Krukenberg V, Riedel D, Tegetmeyer HE, Boetius A. (2015). Intercellular wiring enables electron transfer between methanotrophic archaea and bacteria. Nature 526: 587-590.

Whelan S, Goldman N. (2001). A general empirical model of protein evolution derived from multiple protein families using a maximum-likelihood approach. Mol Biol Evol 18: 691-699.

Xiao K, Malvankar NS, Shu C, Martz E, Lovley DR, Sun X. (2016). Low energy atomic models suggesting a pilus structure that could account for electrical conductivity along the length of Geobacter sulfurreducens pili. Sci Rep 6: 23385.

Yi H, Nevin KP, Kim B-C, Franks AE, Klimes A, Tender LM et al. (2009). Selection of a variant of Geobacter sulfurreducens with enhanced capacity for current production in microbial fuel cells. Biosens Bioelectron 24: 3498-3503.

Supplementary Information accompanies this paper on The ISME Journal website (http://www.nature.com/ismej) 